\title{
Dark-field microscopy enhances visibility of CD31 endothelial staining
}

\author{
Eva Jennische, ${ }^{1}$ Stefan Lange, ${ }^{2}$ Ragnar Hultborn ${ }^{3}$
}

${ }^{1}$ Department of Medical Chemistry and Cell Biology, Institute of Biomedicine, Sahlgrenska Academy, University of Gothenburg

${ }^{2}$ Department of Microbiology and Immunology, Institute of Biomedicine, Sahlgrenska Academy, University of Gothenburg

${ }^{3}$ Department of Oncology, Institute of Clinical Sciences, Sahlgrenska Academy, University of Gothenburg, Sweden

\begin{abstract}
A simple dark field microscopy technique was used for visualization of blood vessels in normal human renal tissues and carcinoma. Phase contrast condenser ring apt for high power objectives was combined with a 10x objective in order to create a dark field illumination of the specimens examined. The endothelial lining of the vessels had been stained by using CD31 monoclonal antibodies combined with conventional peroxidase immunohistochemistry. The final DAB addition used for this technique induced an intense light scatter observable in the dark field microscope. This scattered light originating from the endothelial lining made the walls of the bright vessels easily detectable from the dark background.
\end{abstract}

Key words: Dark-field microscopy; immunohistochemistry; CD31.

Correspondence:Eva Jennische, Department of Medical Chemistry and Cell Biology, Institute of Biomedicine Sahlgrenska Academy, University of Gothenburg, Box 440, SE-405 30 Gothenburg, Sweden.

E-mail: eva.jennische@anatcell.gu.se

Contributions: All the authors contributed equally to planning, performing and writing. All the authors have read and approved the final version of the manuscript and agreed to be accountable for all aspects of the work.

Conflict of Interest: None of the authors have any conflict of interest related to this work. 


\section{Introduction}

Vascular architecture is critical for normal and neoplastic tissue function. Mapping of the vessels in the different tissues is commonly performed with immunohistochemical, peroxidase targeting with CD31 monoclonal antibodies against this specific endothelial epitope. The evaluation of the resulting brown pigment may be subjective, and digital image analysis is frequently hampered by low contrast. The visibility of the peroxidase induced DAB-protein precipitate can, however, be intensified and consequently easily detectable by its ability to scatter light when using the dark field microscopic technique. ${ }^{1}$ In this technique a special condenser system prevents direct light from the light source to reach the objective. Thus, only diffracted light in the specimens will be observed. In the present study we show that the same dark field effect can be obtained using a standard microscope equipped with a phase contrast condenser.

\section{Materials and Methods}

\section{Tissues}

Post-nephrectomy human kidneys, including cortical, medullary tissues, and clear cell carcinoma, were fixed in $4 \%$ formalin. Paraffin embedding followed and the specimens were finally sectioned at a thickness of 4 $\mu \mathrm{m}$. The specimens were retrieved from routine clinical pathology.

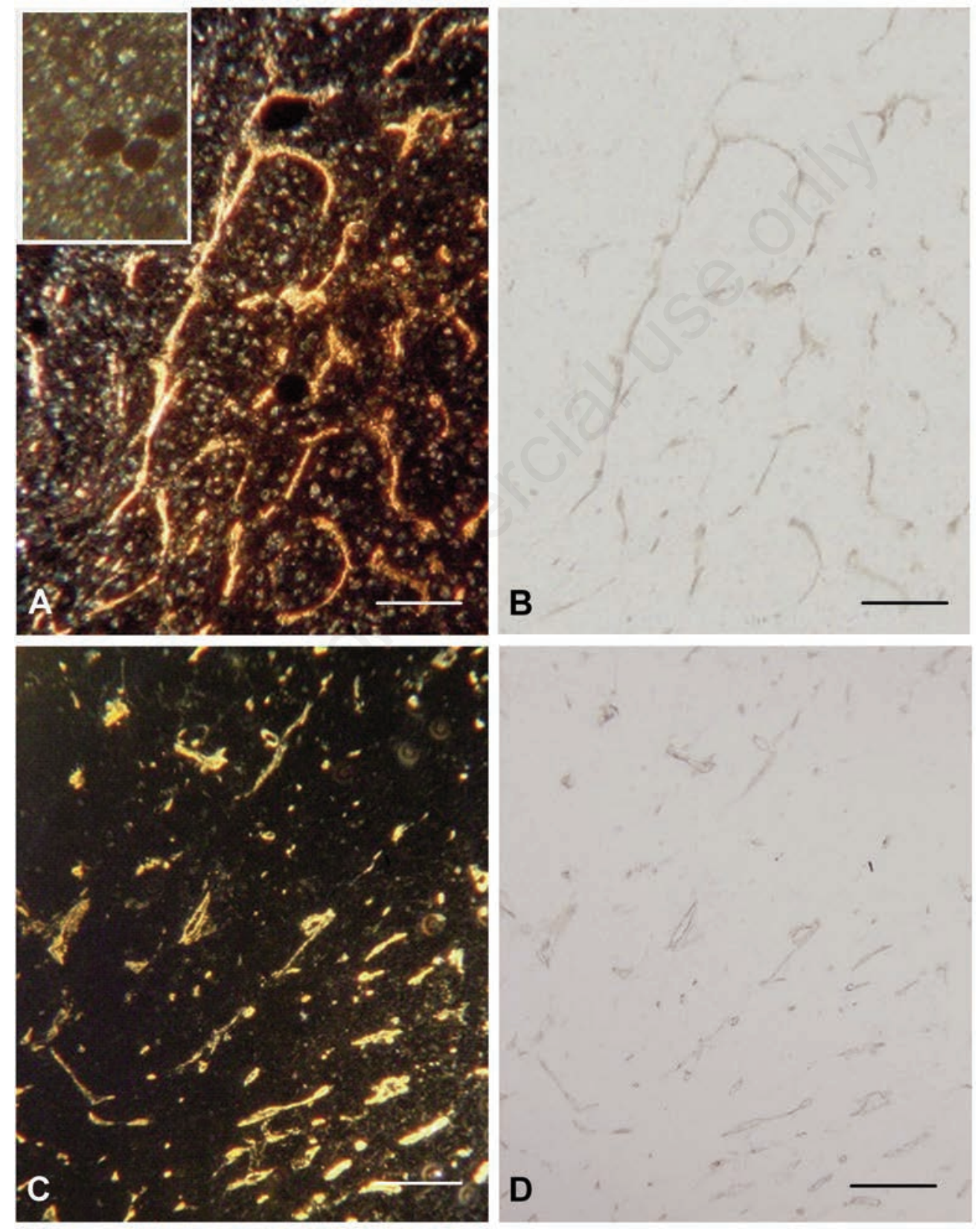

Figure 1. Renal carcinoma, $4 \mu \mathrm{m}$ paraffin sections. Left panel dark field, right panel bright field. A) Dark-field 10x Ph condensor 3 of a section counterstained with haematoxylin also visualizing cell nuclei. Insert, dark-field of an adjacent section. IHC prepared as above but not DAB exposed. B) Same area as A, in bright-field. C) Renal carcinoma not counterstained with haematoxylin in dark-field. D) Same area in bright-field. Scale bars: $100 \mu \mathrm{m}$. 


\section{CD31 immunohistochemistry}

The sections of the paraffin-embedded samples were cut and mounted on Superfrost ${ }^{\circledR}$ Plus glasses (Menzel GmbH \& Co. KG), deparaffinised, hydrated and treated with a solution of 3\% hydrogen peroxide which quenches endogenous peroxidase activity. Antigen retrieval was performed by heating the sections for $15 \mathrm{~min}$ at $95^{\circ} \mathrm{C}$ in a TRIS/EDTA buffer $\mathrm{pH} 8$ using a Decloaking Chamber ${ }^{\mathrm{TM}} \mathrm{NxGen}$ (BIOCare Medical, Pacheco, CA, USA). After blocking with $2.5 \%$ normal goat serum the sections were incubated at $5^{\circ} \mathrm{C}$ overnight with a rabbit monoclonal antibody against $\mathrm{CD} 31$ (ab182981; Abcam, Cambridge, UK) at 1/4500 dilution in PBS. Anti-rabbit impress-HRP (Vector Labs, Burlingame, CA, USA) was used as secondary reagent and the immunoreactions were visualized by using Liquid DAB+ substrate chromogen system (Dako, Glostrup, Denmark) giving a particular brown reaction product. In some sections the cell nuclei were counterstained with haematoxylin. After dehydration the sections were mounted in DPX (Sigma-Aldrich, St. Louis, MO, USA).
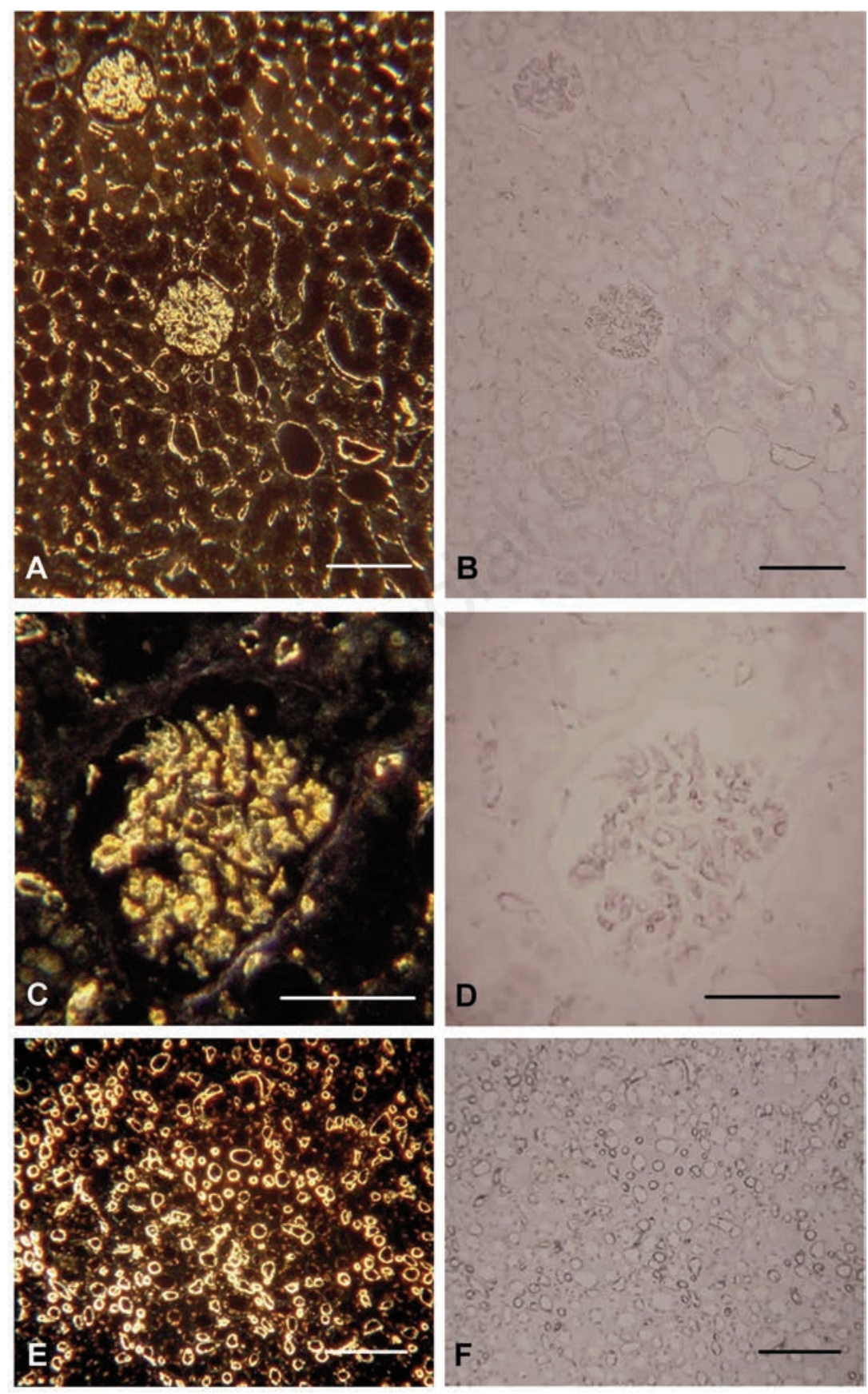

Figure 2. Human kidney, 4 um paraffin sections. Left panel dark field, right panel bright field. A) Renal cortex including glomeruli and tubuli, dark-field 10x Ph condensor 3. B) Same area in bright field. C) Dark-field 32x with dedicated dark-field condensor including substage immersion oil. D) Same area in bright-field. E) Renal medulla dark-field 10x Ph condensor 3. F) Same area in bright-field. Scale bars: A,B,E,F) $100 \mu \mathrm{m}$; C,D) $50 \mu \mathrm{m}$. 


\section{Dark-field microscopy}

A Zeiss microscope (Standard 16 with a flip-out circular phase contrast condenser Ph1, Ph2, Ph3) was used. A 10x objective (irrelevant whether phase contrast or not) was used and the condenser was set "Ph3" for high power objectives, blocking direct light from the phase ring to pass the low power objective. The set-up is similar to that described by Antolović et al. ${ }^{2}$ A series of control sections were used, namely:

a) unstained;

b) exposed to the primary antibody only;

c) exposed as b plus the secondary HRP-antibody;

d) as c plus $\mathrm{H}_{2} \mathrm{O}_{2}$ /diaminobenzidine (DAB).

In a second series the sections a to $\mathrm{d}$ were counterstained with haematoxylin.

\section{Results and Discussion}

The results showed that the light scattering effects was caused by the DAB precipitate, since only section $d$ above gave a positive signal in the blood vessels. Sections a to c were negative. In the sections stained with haematoxylin the staining gave some light scattering (Figure 1A).

The use of the two microscopic techniques bright field $v s$ dark field is demonstrated in Figures 1 and 2. The endothelial linings of the blood vessels in human kidney cortex, including glomeruli and peritubular capillaries and in the endothelial cells of the vasa recta in the medulla are visualized by the intense light scatter emitted from the DAB precipitate (Figure 2). Figure 1 illustrates capillaries in a renal carcinoma. In the set of illustrations where the sections have been counterstained with haematoxylin it is clear that stained cell nuclei also disperse light. It is also shown that DAB exposure is required to produce the light scattering precipitate.

The results demonstrate that the illuminated endothelial cells are easily detectable from the dark background by use of the dark field microscopic technique. The bright field technique, visualizes the brownish pigment in the endothelial lining with a lower contrast against the background. These results tentatively suggest the use of the dark field microscopic technique for a future use in the art of digital image analysis.
We used a combination of a phase contrast condenser apt for high power objectives "Ph3" combined with a 10x objective. This was done since dark field condensers are not frequently used today. The magnification obtained with this technique is often enough for visualizing the vascular network, but if higher-power objectives are required a dark-field condenser including use of substage oil immersion must be used. Dark-field microscopy, for a long time rarely used, has recently been revived for imaging nanoparticle labelled bacteria ${ }^{3}$ and molecular transformations in cell biology, ${ }^{4}$ thus the technique might have a renaissance in biological research.

We believe that this simple enhancement of vascular visibility might add to easier visual and/or to quantitative description of vascular density of relevance in e.g. tumour malignancy grading. The present results achieved by the use of the dark-field microscopy in combination with peroxidase immunohistochemistry suggest a future use of this technique.

\section{Acknowledgements}

The study was supported by the King Gustav V Jubilee Clinic Research Foundation.

\section{References}

1. Fan L, Tian Y, Yin R, Lou D, Zhang X, Wang M, et al. Enzyme catalysis enhanced dark-field imaging as a novel immunohistochemical method. Nanoscale 2016; 8: 8553-8.

2. Antolović, V, Marinović M, Filić V, Weber I. A simple optical configuration for cell tracking by dark-field microscopy. J Microbiol Methods 2014;104:9-11.

3. Xu H, Tang F, Dai J, Wang C, Zhou X. Ultrasensitive and rapid count of Escherichia coli using magnetic nanoparticle probe under dark-field microscope. BMC Microbiol 2018;1241-5.

4. Wang B, Sun D, Zhang C, Wang K, Bai J. Dark-field microscopy for characterization of single molecule dynamics in vitro and in vivo. Anal Methods 2019;11:2778-84.

Received for publication: 25 March 2020. Accepted for publication: 17 June 2020.

This work is licensed under a Creative Commons Attribution-NonCommercial 4.0 International License (CC BY-NC 4.0).

CCopyright: the Author(s), 2020

Licensee PAGEPress, Italy

European Journal of Histochemistry 2020; 64:3133

doi:10.4081/ejh.2020.3133 